\title{
Treatment of hyperlipidemic acute pancreatitis with modified Dachengqi decoction combining with conventional therapy based on "six-hollow-organs to be unblocked" theory
}

\author{
Guoxiong Liu ${ }^{1}$, Fang Liu ${ }^{1}$, Long Xiao ${ }^{1}$, Qiaogui Kuang ${ }^{2}$, Xingfeng $\mathrm{He}^{1}$, Yu Wang ${ }^{3}$, Yang Yu ${ }^{3}$ \\ ${ }^{1}$ Department of Orthopedics, the First Affiliated Hospital of Guizhou University of Traditional Chinese Medicine, Guiyang, China; ${ }^{2}$ College of \\ Acupuncture and Orthopedics, Guizhou University of Traditional Chinese Medicine, Guiyang, China; ${ }^{3}$ Puding County Hospital of Traditional \\ Chinese Medicine, Anshun, China \\ Contributions: (I) Conception and design: G Liu, F Liu; (II) Administrative support: L Xiao; (III) Provision of study materials or patients: Q Kuang; (IV) \\ Collection and assembly of data: X He, Y Wang; (V) Data analysis and interpretation: G Liu, F Liu; (VI) Manuscript writing: All authors; (VII) Final \\ approval of manuscript: All authors. \\ Correspondence to: Guoxiong Liu. Department of Orthopedics, the First Affiliated Hospital of Guizhou University of Traditional Chinese Medicine, \\ Guiyang, China. Email: zgliuguoxiong@163.com.
}

Background: The increase of triglyceride (TG) can induce coronary heart disease, atherosclerosis, pancreatitis and other diseases and is the most common inducing factor of acute pancreatitis (AP) second only to biliary tract disease and drinking. The pathogenesis of hyperlipidemic acute pancreatitis (HLAP) is not exactly clear, but it may be related to the toxic effect of the increase of free fatty acids produced by TG decomposition on the pancreas itself, microcirculation disorder of the pancreas, and calcium overload. At present, non-surgical therapy is the main treatment for HLAP. The key to preventing recurrence is to reduce blood lipids, change the diet structure, and reduce weight. This study aimed to treat HLAP with modified Dachengqi decoction combined with conventional therapy, based on the "six-hollow-organs to be unblocked" theory.

Methods: Forty patients with HLAP who received treatment in the First Affiliated Hospital of Guizhou University of Traditional Chinese Medicine and Puding County Hospital of Traditional Chinese Medicine from September 2016 to August 2019 were selected and divided into a control group and an intervention group, each with 20 cases, following a random number table. The control group was treated with conventional therapy while the intervention group was treated with modified Dachengqi decoction combined with conventional therapy.

Results: After treatment, the cure rate and the total effective rate were $60 \%$ and $95 \%$ respectively in the intervention group, and $25 \%$ and $75 \%$ respectively in the control group $(\mathrm{P}<0.05)$. The TG, serum amylase, leukocyte count, and neutrophil ratio of the two groups decreased significantly after treatment, and there was a greater decrease in the intervention group than that in the control group, with this being significantly different between the two groups. The gastrointestinal function score, total score of the acute physiology and chronic health evaluation II (APACHE II), and the pain score of the visual analog scale (VAS) decreased markedly in the two groups after the treatment, with scores in the intervention group being significantly lower than those in the control group $(\mathrm{P}<0.05)$.

Conclusions: Modified Dachengqi decoction combined with conventional therapy has a better therapeutic effect on HLAP than conventional therapy.

Keywords: Hyperlipidemic acute pancreatitis (HLAP); etiology and pathogenesis; modified Dachengqi decoction; "six-hollow organs to be unblocked" theory

Submitted Apr 20, 2020. Accepted for publication Jun 24, 2020.

doi: 10.21037/apm-20-1106

View this article at: http://dx.doi.org/10.21037/apm-20-1106 


\section{Introduction}

High triglyceride (TG) levels can lead to serious diseases, including coronary heart disease, atherosclerosis, pancreatitis, etc. (1). Hyperlipidemia is the most common inducer of acute pancreatitis (AP) second only to biliary tract disease and drinking. Studies from China show that hyperlipidemic AP (HLAP) accounts for about $8.2-12.6 \%$ of AP, with the incidence rate increasing each year (2). Its pathogenesis is uncertain and may be related to the toxic effect of increased free fatty acids produced by TG decomposition in the pancreas itself, microcirculation disorder of the pancreas, and calcium overload. The symptoms include persistent upper abdominal or left upper abdominal pain, abdominal distention, or vomiting. One study found that mild HLAP could be treated by nonsurgical therapy, but the effect is uncertain (3). The key to preventing recurrence is quickly reducing blood lipid, changing diet structure, and reducing weight (4). One of the research directions of HLAP involves exploring a classical formula that can rapidly reduce blood lipid levels. In nowadays, blood purification including hemofiltration, blood perfusion and plasma exchange is the main method to lower blood lipid level in short time. But these methods are all expensive. Dachengqi decoction's main effects include lowering blood lipid levels, enabling earlier enteral nutrition, improving gastrointestinal function, inhibiting pro-inflammatory factors, improving microcirculation, performing coagulation functions of the pancreas, among other benefits. On the basis of the abovementioned studies, this study combined conventional therapy with Dachengqi decoction for the treatment of HLAP. We present the following article in accordance with the STROBE reporting checklist (available at http://dx.doi.org/10.21037/ apm-20-1106).

\section{Methods}

The study was approved by the Ethic committee of the First Affiliated Hospital of Guizhou University of Traditional Chinese Medicine, and was conducted in accordance with the Declaration of Helsinki (as revised in 2013). Because of the retrospective nature of the research, the requirement for informed consent was waived.

\section{Study objects}

Forty patients with HLAP who received treatment in the First Affiliated Hospital of Guizhou University of TCM and
Puding County Hospital of Traditional Chinese Medicine from September 2016 to August 2019 were selected and divided into two groups of 20 cases each, following a random number table. The inclusion criteria were as follows: (I) patients who met the Western medical diagnostic criteria of HLAP and were dialectically classified with the "syndrome of dampness and heat of liver and gall", the "syndrome of heat deposition in the hollow organs", or the "syndrome of toxin accumulation and blood stasis"; (II) patients aged between 18 and 65 years old; (III) patients who could understand this study; (IV) patients without severe heart, liver, kidney, or other functional deficiencies; (V) patients without a history of digestive system operation or local complications of the digestive system in 1 year. The exclusion criteria were as follows: (I) those who were mistakenly included; (II) patients younger than 18 years old or older than 65 years old; (III) patients who could tolerate the course of treatment; (IV) those who did not strictly comply with the regulations or who took other therapies such as oral drugs; (V) those who do not follow the treatment requirements and had incomplete data that would have affected the judgment of curative effect. All patients signed informed consent.

\section{Diagnostic standards}

The primary diagnostic criteria of $\mathrm{AP}$ are the following (5): having abdominal pain in accordance with AP (sudden, acute, severe, persistent upper abdominal pain, radiating to the back), and two items of three items described as follows: (I) lipase activity and/or serum amylase three times higher than the upper limit of the normal value; (II) AP change in enhanced computed tomography (CT), magnetic resonance imaging (MRI), or abdominal ultrasonography; (III) TG greater than $11.3 \mathrm{mmol} / \mathrm{L}$, or chylemia with TG being within the normal range.

Traditional Chinese medicine (TCM) diagnosis and syndrome differentiation was conduced according to the "Expert Consensus on TCM Diagnosis and Treatment Acute Pancreatitis" (6) issued by the Spleen and Stomach Disease Branch of the China Association of Chinese Medicine in 2017. According to these definitions, HLAP can be divided into five types: dampness and heat syndrome of the liver and gallbladder, stagnation of liver Qi, liver stagnation and spleen deficiency syndrome, syndrome of heat deposition in the hollow organs, and syndrome of toxin accumulation and blood stasis. Modified Dachengqi decoction is mainly suitable for the patients with dampness and heat syndrome of the liver and gallbladder, syndrome of 
heat deposition in the hollow organs, and syndrome of toxin accumulation and blood stasis.

\section{Methodology}

\section{Medical treatment}

Twenty cases in the control group received conventional treatment which comprised the following: (I) fasting and water deprivation and gastrointestinal decompression; (II) spasmolysis and pain relief; (III) inhibiting gastric acid secretion, improving microcirculation, balancing the internal environment, and rectifying water and electrolyte disorder based on clinical need; (IV) early parenteral nutrition support and gradual transition from liquid food to general food after remission; (V) control and prevention of infection. Twenty cases in the intervention group received not only the conventional treatment of the control group but also orally administered modified Dachengqi decoction composed of roasted rhubarb $15 \mathrm{~g}$, bupleurum $10 \mathrm{~g}$, Fructus aurantii $10 \mathrm{~g}$, Magnolia officinalis Rehd.et Wils $10 \mathrm{~g}$, Cinnamomum cassia Presl $15 \mathrm{~g}$, Poria cocos 12 g, Paeonia suffruticosa Andr. $15 \mathrm{~g}$, peach kernel $7 \mathrm{~g}$, safflower $7 \mathrm{~g}$, earthworm $20 \mathrm{~g}$, Alisma orientalis $15 \mathrm{~g}$, Polygonum cuspidatum $20 \mathrm{~g}$, chicken gizzard membrane $12 \mathrm{~g}$, Ligusticum chuanxiong Hort $15 \mathrm{~g}$, hawthorn $10 \mathrm{~g}$, and liquorice $3 \mathrm{~g}$. The above drugs were added and subtracted according to the syndrome differentiation of traditional Chinese medicine. It was boiled with water twice, then mixed together, divided into two equal parts, and taken two times, at one dose per day.

\section{Observation indexes}

The observation indexes included blood amylase level, serum TG, and gastrointestinal function scores.

\section{Clinical efficacy criteria}

Recovery standards were determined according to the Guiding Principles of Clinical Research on New Drugs of Chinese Medicines (7) and the modified Criteria of Diagnosis and Therapeutic Effect of Syndromes in Traditional Chinese Medicine (8). The criteria for judging the clinical treatment effect for recovery, significant improvement, effectiveness, and ineffectiveness are as follows:

* Recovery: the symptoms and signs disappear, the laboratory indexes return to the normal range, the imaging examinations completely return to normal, and the complications completely disappear.

* Significant improvement: symptoms and signs basically disappear, laboratory indexes improve significantly though some indexes are not within the normal range, imaging examinations basically return to normal, complications basically disappear, and acute physiology and chronic health evaluation II (APACHE II) score decreases by more than $50 \%$.

* Effectiveness: the symptoms and signs improve, the relevant auxiliary examination indexes and imaging examination including CT are somewhat recovered compared with that at the admission but have not returned to normal, and APACHE II score decrease by less than $50 \%$.

* Ineffectiveness: no change in clinical symptoms and signs, conversion to surgery, or an APACHE score reduction lower than $30 \%$.

* Death: death due to rapid aggravation of the disease.

Visual analogue scale (VAS) pain scoring (9): patients' pain degree was evaluated from 0 to 10 points with VAS into four levels: 0 represented no pain and no discomfort; 1 to 3 represented patients having mild pain but with no impact on life and work; 4 to 6 represented patients having moderate pain with life and work partially affected; and 7 to 10 represented patients having severe pain with life and work seriously affected, or no ability to self-care; the higher the score, the more severe the pain the patient suffered.

Gastrointestinal function score and Apache II total score evaluation (10): the gastrointestinal function score was evaluated with the gastrointestinal failure scoring system using the two aspects: abdominal pressure and intestinal nutrition. Scoring ranged from 0 to 4 points: 0 represented normal; 1 represented no enteral nutrition or enteral nutrition less than $50 \% 3$ days after treatment; 2 represented no enteral nutrition or existence of abdominal hypertension; 3 represented the coexistence of absent enteral nutrition and abdominal hypertension; and 4 represented the existence of abdominal compartment syndrome. The higher the score was, the more serious the disease. APACHE II is based on four major items: age, chronic health status, Glasgow coma scale (GCS), and acute physiology indexes. Age was scored as follows: under 45 years old, $0 ; 45-54$ years old, 2 ; 55-64 years old, 3; 65-74 years old, 5; and over 74 years old, 6. Chronic health status was scored as follows: none, 0 ; elective surgery, 2; emergency surgery, 5. GCS score is equal to the sum of the following 3 items: eye opening response, 4 points at most; language response, 5 points at most; exercise response, 6 points at most. The final score = 15-GCS. Acute physiological indexes included 11 items such as body temperature, blood pressure, heart rate, respiratory rate, oxygenation, arterial $\mathrm{pH}$, serum sodium 
Table 1 Comparison of healing rate

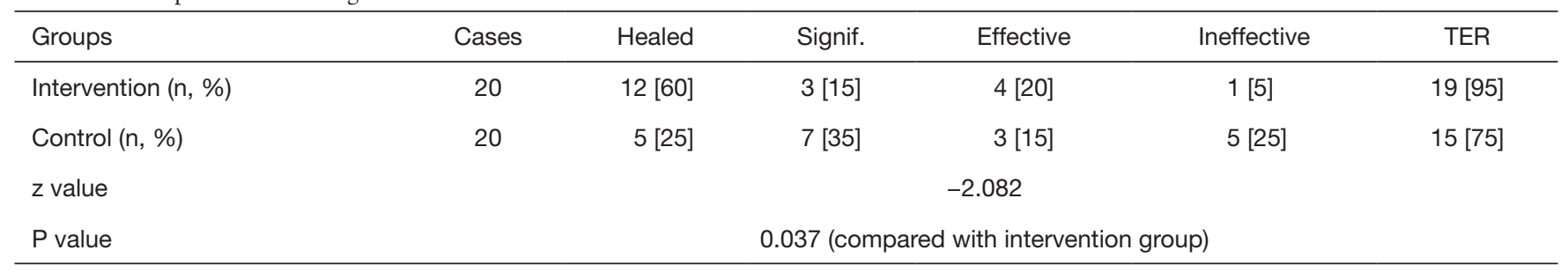

Signif., significantly effective; TER, total effective rate.

Table 2 Comparison of the serum amylase and triglyceride levels between the two groups

\begin{tabular}{lccccc}
\hline \multirow{2}{*}{ Groups } & \multicolumn{2}{c}{ Serum amylase $(\mathrm{U} / \mathrm{L})$} & \multicolumn{2}{c}{ Serum triglyceride $(\mathrm{mmol} / \mathrm{L})$} \\
\cline { 2 - 3 } \cline { 5 - 6 } Intervention & Before treatment & After treatment & & Before treatment & After treatment \\
Control & $790.26 \pm 71.76$ & $237.04 \pm 43.32$ & $15.59 \pm 2.95$ & $2.53 \pm 1.96$ \\
$\mathrm{t}$ & $774.33 \pm 76.28$ & $592.17 \pm 38.62$ & $12.56 \pm 2.15$ & $7.48 \pm 1.90$ \\
$\mathrm{P}$ & 0.145 & -6.246 & 1.058 & -7.006 \\
\hline
\end{tabular}

and potassium, creatine, hematocrit, and white blood cell count with the lowest point score being 0 , representing normal, and the highest score being 4 . The greater the deviation from the normal status, the higher the score. The APACHE II score is the sum of four major items. It is a scoring system of the whole body: the lower the score, the better the body's function is; the higher the score, the worse the body's function is.

\section{Statistical analysis}

SPSS20.0 statistical software was used to analyze and process the data. The measurement data was expressed by $(\mathrm{x} \pm \mathrm{s})$. For the self-control comparison between before and after the treatment, the paired sample $t$-test was used. Two independent samples $t$-test was used for the comparison between two groups. Wilcoxon rank-sum test was used to compare the clinical efficacy, and the difference was statistically significant when $\mathrm{P}<0.05$.

\section{Results}

\section{Comparison of the baseline data between the two groups}

In the intervention group, 20 cases were collected, including 12 males and 8 females, aged from 18 to 50 years $(39.3 \pm 3.6$ years). In the control group, a total of 20 cases were collected, including 13 males and 7 females, aged from 18 to 50 years $(40.8 \pm 4.4$ years). There was no significant difference in age and gender between the two groups $(\mathrm{P}>0.05)$.

\section{Comparison of healing rate between the two groups}

The effective rate in the intervention group and the control group was $95 \%$ and $75 \%$ respectively, and the difference was statistically significant $(\mathrm{P}<0.05)$ (Table 1).

\section{Comparison of serum amylase, serum TG, and inflammatory index between the two groups}

There was no significant difference in the serum amylase and serum TG levels before the treatment between the intervention group and the control group $(\mathrm{P}>0.05)$. All indexes of the two groups were improved after treatment compared with those before treatment, but compared with that of the control group, the level of serum amylase and TG in the intervention group was significantly improved $(\mathrm{P}<0.05)$ (Table 2).

\section{Comparison of gastrointestinal function score and APACHE II total score between the two groups}

There was no significant difference in gastrointestinal function score and APACHE II total score between the 
Table 3 Comparison of gastrointestinal function score, APACHE II total score, and VAS pain score

\begin{tabular}{lccccccc}
\hline \multirow{2}{*}{ Groups } & \multicolumn{2}{c}{ Gastrointestinal function score } & & \multicolumn{2}{c}{ APACHE II total score } & & \multicolumn{2}{c}{ VAS pain score } \\
\cline { 2 - 3 } & Before treatment & After treatment & & Before treatment & After treatment & Before treatment & After treatment \\
\hline Intervention & $1.80 \pm 0.41$ & $0.05 \pm 0.22$ & & $142.85 \pm 15.67$ & $41.15 \pm 15.29$ & $6.85 \pm 2.18$ & $1.25 \pm 0.62$ \\
Control & $2.10 \pm 0.21$ & $0.42 \pm 0.51$ & & $141.75 \pm 7.79$ & $52.33 \pm 12.53$ & $7.75 \pm 1.14$ & $2.15 \pm 1.42$ \\
$\mathrm{t}$ & -1.677 & -2.80 & & 0.226 & -2.136 & -1.32 & -2.46 \\
$\mathrm{P}$ & 0.104 & 0.009 & 0.823 & 0.041 & 0.197 & 0.02 \\
\hline
\end{tabular}

VAS, visual analogue scale.

Table 4 Comparison of the white blood cell count and neutrophil count

\begin{tabular}{lcccc}
\hline \multirow{2}{*}{ Groups } & \multicolumn{2}{c}{ White blood cell count $\left(\times 10^{9} / \mathrm{L}\right)$} & \multicolumn{2}{c}{ Neutrophil count $(\%)$} \\
\cline { 2 - 4 } \cline { 4 - 5 } Intervention & Before treatment & After treatment & Before treatment & After treatment \\
Control & $8.43 \pm 3.84$ & $5.86 \pm 1.65$ & $62.05 \pm 21.45$ & $49.80 \pm 16.34$ \\
$\mathrm{t}$ & $10.82 \pm 4.64$ & $9.96 \pm 4.83$ & $72.38 \pm 22.45$ & $65.60 \pm 13.28$ \\
$\mathrm{P}$ & -1.574 & -2.845 & 1.621 & 2.161 \\
\hline
\end{tabular}

intervention group and the control group before the treatment $(\mathrm{P}>0.05)$. The scores after the treatment in the two groups were lower than that before treatment, but compared with those in the control group, the gastrointestinal function score, APACHE II total score, and VAS pain score in the intervention group decreased more significantly $(\mathrm{P}<0.05)($ Table 3$)$.

\section{Comparison of inflammatory indexes between the two groups}

There was no significant difference in white blood cell count and neutrophil count between the intervention group and the control group before treatment $(\mathrm{P}>0.05)$. These indicators in the two groups were improved after the treatment compared with those before the treatment, and the differences were statistically significant $(\mathrm{P}<0.05)$. Compared with those in the control group, the white blood cell count and neutrophil count in the intervention group improved more significantly after the treatment, and the differences were statistically significant $(\mathrm{P}<0.05)$ (Table 4).

\section{Syndrome differentiation results and treatment outcome of TCMs}

After treatment, abdominal pain has been relieved and appetite improved in most patients. The sign of tongue and color of urine both turned to be better than those before treatment. Details were listed in Table 5 .

\section{Discussion}

At present, the specific mechanism of HLA is not clear, but it is considered to be related to the following factors (11-13): (I) free fatty acid accumulation in the pancreas that exceeds the load of the pancreas' processing ability, directly causing cell and capillary damage and resulting in corresponding tissue necrosis and the entrance of toxic substances into the blood circulation; (II) the TGs in and around the pancreas decomposing into a large number of free fatty acids, which activate trypsinogen and cause the pancreas to selfdigest; (III) a high concentration of TGs inducing systemic inflammatory response; (IV) a high level of TG activating the phospholipidation of cells, causing cell membrane damage and releasing a large number of enzymes, thus aggravating self-digestion. The clinical manifestations of HLAP include vomiting, nausea, abdominal distention, fever, acute epigastric pain, enteroparalysis resulting in cessation of anal discharge and defecation, and increase of blood pancreatin $(14,15)$.

Based on the clinical symptoms and signs of traditional Chinese medicine, HLAP can be classified into "abdominal 
Table 5 Treatment outcome of traditional Chinese medicine

\begin{tabular}{lll}
\hline Syndromes & Before treatment & After treatment \\
\hline $\begin{array}{l}\text { Dampness and heat } \\
\text { syndrome of liver and } \\
\text { gallbladder }\end{array}$ & $\begin{array}{l}\text { Epigastric distention pain, sticky stool. Secondary } \\
\text { symptoms: chest distress, fever, thirst-inducing } \\
\text { drink, short yellow urine; red tongue, yellow greasy normal or slightly yellow urine; slightly red tongue with } \\
\text { or thin yellow fur; rapid and stringy pulse }\end{array}$ & $\begin{array}{l}\text { poor appetite, no thirst, no fever, normal bowel movement, } \\
\text { thin and white fur, or red tip of the tongue with greasy fur, } \\
\text { or slightly toothed tongue body; a gentle and powerful, } \\
\text { slightly rapid pulse }\end{array}$ \\
$\begin{array}{l}\text { Syndrome of heat } \\
\text { deposition in the hollow } \\
\text { organs }\end{array}$ & $\begin{array}{l}\text { Abdominal distention and pain, refusal to press, } \\
\text { dry stool, afternoon tidal fever, chest fullness, } \\
\text { vomiting, bad breath; lower-volume, dark yellow }\end{array}$ & $\begin{array}{l}\text { Abdominal pain obviously relieved or disappeared. No } \\
\text { poor appetite, soft abdomen, no thirst, no fever, normal } \\
\text { feces and urine or slightly dry stool or slightly yellow urine; } \\
\text { slippery pulse }\end{array}$ \\
$\begin{array}{l}\text { Syndrome of toxin } \\
\text { accumulation and blood } \\
\text { stasis }\end{array}$ & $\begin{array}{l}\text { Abdominal tingling, refusal to press, fixed pain, dry } \\
\text { stool, restlessness, bruises with ecchymosis, fever, no poor appetite, no thirst, no fever, normal feces and } \\
\text { dark-yellow urine; red tongue or with ecchymosis } \\
\text { on the tongue; stringy or astringent pulse }\end{array}$ & $\begin{array}{l}\text { urine or slightly yellow urine; light red tongue with thin and } \\
\text { white fur or red tip of tongue; gentle and powerful pulse }\end{array}$ \\
\hline
\end{tabular}

pain", "stomach heartache", "spleen heartache", and "abdominal fullness pain" and other syndromes (16). It is suggested that the treatment of "abdominal fullness pain should be treated with Dachengqi decoction". The etiology and pathogenesis of the disease are complicated. It may be Yangming hollow organ excess syndrome caused by a disharmony of diet, stagnation of liver Qi, stagnation of Qi and blood, and heat deposition which burns body fluid, or mutual stagnation syndrome of heat and toxins resulting from stagnation of the liver Qi, accumulation of damp heat in the liver and gallbladder, blockage of the middle Jiao, stagnation of dampness and heat in the spleen, distribution and metabolism disorder of body fluids, and toxin formation from accumulation and stagnation of body fluid. The treatment strategy should be made based on an understanding of the etiology and pathogenesis, and the characteristics of symptoms in different periods of the disease (17), with the goal of rapidly reducing blood lipids. We used a modified Dachengqi decoction, in which rhubarb is the dominant ingredient and effectuates a purging of heat and defecation, and clears the gastrointestinal stasis. Glauber's salt can soften hardness and moisten dryness, and help the rhubarb to dredge the hollow organs. Magnolia officinalis Rehd.et Wils can widen the intestines and lower the $\mathrm{Qi}$, and remove the stagnation and distention to cure fullness. Aurantii fructus immaturus can remove the accumulation of Qi, regulate the Qi circulation, eliminate the fullness, remove invisible Qi stagnation, and strengthen the purgation effect of rhubarb and Glauber's salt. It has been reported that Dachengqi decoction can start enteral nutrition early and improve gastrointestinal function; inhibit pro-inflammatory factors such as INF- $\alpha$, IL- $1 \beta$, IL6 , and IL-8; regulate the immune inflammatory response; and improve the microcirculation and coagulation function of the pancreas (18). It was also found that Dachengqi decoction has the effect of lowering blood lipid levels. Rhubarb, as main ingredient of the prescription, can inhibit the expression of the PPARG gene in fat formation pathway to reduce the generation of PPARG, and then reduce serum TG and free fatty acid levels $(19,20)$. Since INF- $\alpha$ has positive regulatory effect on PPARG, inhibition of INF- $\alpha$ can reduce blood lipids through negative feedback regulation (21). Bupleurum has heat dissipation, liver-soothing, and depression-relieving functions, while saikosaponin can reduce TG, accelerate TC excretion with feces, reduce TC concentration, and effectively prevent the formation and development of hyperlipidemia (22). Poria cocos can strengthen the spleen and calm the nerves. This mushroom's polysaccharide has the function of liver protection, immune and leukocyte regulation, improvement of the hematopoietic system, a restoring the function of tissue cells (23). Cinnamomum cassia Presl, Prunus persica (L.) Batsch, and earthworm can calm Qi, promote blood circulation, relieve constipation, and relieve pain. Modern pharmacology has found that the ingredients can regulate IL-2 and IL-4 secretion and stimulate TNF- $\alpha$ to play an anti-inflammatory and immunomodulatory role (24). Chicken gizzard membrane is used to treat dyspepsia and food stagnation, and to strengthen the spleen and stomach. Modern pharmacology shows that chicken gizzard 
membrane can increase intestinal peristalsis; promote intestinal emptying; and reduce blood sugar, TG, and fat accumulation in the liver and mesentery (25). Safflower and Ligusticum chuanxiong Hort can activate the Qi and blood circulation, disperse blood stasis, and relieve pain. It has been reported that they have the function of improving blood circulation of ischemic tissue and promoting microcirculation $(26,27)$. Alisma orientalis and hawthorn have the effects of strengthening the stomach, moistening, promoting Qi, resolving turbidity, and reducing blood fat. Polygonum cuspidatum and Paeonia suffruticosa Andr. have the functions of cooling the blood, promoting blood circulation, and removing blood stasis. It was found that they can jointly reduce blood fat by reducing the amount of TC, TG and LDL-C in the serum of patients with hyperlipidemia and by increasing high-density lipoprotein cholesterol (HDL-C) (28-30). It was also demonstrated that Polygonum cuspidatum has protective effects on human organs (31). Finally, Glycyrrhiza has the effect of harmonizing various drugs.

It is generally believed that "routine therapy combined with traditional Chinese medicine can significantly improve the clinical efficiency of HLAP (32)". Based on the "sixhollow-organs to be unblocked" theory and the functions of Dachengqi decoction in unblocking the hollow organs, influencing pathogenic factors, removing fever and dampness, and relieving pain, Dachengqi Decoction was applied to treat HLAP with dampness and heat syndrome of the liver and gallbladder, syndrome of heat deposition in the hollow organs, or syndrome of toxin accumulation and blood stasis, leading to obvious curative effect. It was found that Dachengqi decoction can excite the intestine; increase gastrointestinal peristalsis, blood flow, smooth muscle excitability, and motilin release (33); eliminate fatty stool; reduce the content of body fat; and thus reduce the level of blood TG and improve the microcirculation of the pancreas.

In recent years, the therapy on HLAP has been continuously improved, especially in traditional Chinese medicine. Its core functions are to reduce or eliminate clinical symptoms, rapidly reduce blood lipids, improve microcirculation, and protect the integrity of endothelial cell structure and function (34). In view of these effects, constant exploration and improvement have been made so as to promote the development of traditional Chinese Medicine and optimize the pattern of modern medical treatment. The sample size of this study is relatively small, and thus a multicenter, large-sample, long-term follow-up study should be performed in the future.

\section{Acknowledgments}

Funding: Guizhou Administration of Chinese Traditional Medicine, QZYY-2019-092; Guizhou Science and Technology Department, QianKeHe LH-2015-7787; Guizhou Science and Technology Department, QianKeHe LH-2015-7800; Guiyang Science and Technology Bureau, 2019-9-2-31.

\section{Footnote}

Reporting Checklist: The authors have completed the STROBE reporting checklist. Available at http://dx.doi. org/10.21037/apm-20-1106

Data Sharing Statement: Available at http://dx.doi. org/10.21037/apm-20-1106

Conflicts of Interest: All authors have completed the ICMJE uniform disclosure form (available at http://dx.doi. org/10.21037/apm-20-1106). The authors have no conflicts of interest to declare.

Ethical Statement: The authors are accountable for all aspects of the work in ensuring that questions related to the accuracy or integrity of any part of the work are appropriately investigated and resolved. The study was approved by the Ethic committee of the First Affiliated Hospital of Guizhou University of Traditional Chinese Medicine, and was conducted in accordance with the Declaration of Helsinki (as revised in 2013). Because of the retrospective nature of the research, the requirement for informed consent was waived.

Open Access Statement: This is an Open Access article distributed in accordance with the Creative Commons Attribution-NonCommercial-NoDerivs 4.0 International License (CC BY-NC-ND 4.0), which permits the noncommercial replication and distribution of the article with the strict proviso that no changes or edits are made and the original work is properly cited (including links to both the formal publication through the relevant DOI and the license). See: https://creativecommons.org/licenses/by-nc-nd/4.0/.

\section{References}

1. Mao E. Intensive management of severe acute pancreatitis. Ann Transl Med 2019;7:687. 
2. Glabay VP, Cridnev OV, Arkharov AV, et al. Complications of the 'open' surgeries for severe acute pancreatitis. Khirurgiia (Mosk) 2017;(10):72-6.

3. Song Y, Zhu H, Du Y. Advances in research of pathogenesis and treatment of hyperlipidemic acute pancreatitis. World Chinese Journal of Digestology 2019;27:112-6.

4. Yin G, Cang X, Yu G, et al. Different Clinical Presentations of Hyperlipidemic Acute Pancreatitis: A Retrospective Study. Pancreas 2015;44:1105-10.

5. Zhao F, Luo S, Mao J, et al. Observation on the therapeutic effect of integrated traditional Chinese and Western Medicine on hyperlipidemia acute pancreatitis. Hebei Medical Journal 2017;17:2659-61.

6. Zhang S, Li H. Expert Consensus on TCM Diagnosis and Treatment Acute Pancreatitis (2017). Chinese Journal of Traditional Chinese Medicine and Pharmacy 2017;32:4085-8.

7. Liang M, Gao T. Investigation and Analysis of Syndromes of Internal Organs in Guideline for Clinical Trials of New Patent Chinese Medicines. Chinese Journal of Basic Medicine in Traditional Chinese Medicine 2008;05:330-1.

8. Criteria of diagnosis and therapeutic effect of internal diseases and syndromes in traditional Chinese medicine (17). Hubei Journal of Traditional Chinese Medicine 2003;11:57.

9. Zhao J, Deng C, Wang M. Study on the clinical effect and safety of acupuncture, massage and traditional Chinese medicine in the treatment of cervical spondylosis. Clinical Research and Practice 2018;05:125-6.

10. Xiong B. Correlation analysis between AP gastrointestinal dysfunction, and CRP, PCT, APACHE II and MCTSI scores. Journal of Hunan Normal University 2018.

11. Zhang X, Lei Y, Luo X. Clinical observation of hemoperfusion combined with Chaishaochengqi decoction in the treatment of hyperlipidemic pancreatitis. China Modern Doctor 2017;04:101-3.

12. Zhang W, Zhao Y, Zeng Y, et al. Hyperlipidemic versus normal lipid acute necrotic pancreatitis: proteomic analysis using an animal model. Pancreas 2012;41:317-22.

13. Chang YT, Chang MC, Su TC, et al. Lipoprotein lipasemutation S447X associated with pancreatic calcification and steatorrhea in hyperlipidemic pancreatitis. J Clin Gastroenterol 2009;43:591-6.

14. Jihui. Clinical observation and nursing of acute pancreatitis. Zhongguo Yiyao Zhinan 2014;24:345-6.

15. Zhou Z, Yang X. Clinical observation of Qingyi Decoction in the treatment of acute pancreatitis. World Latest
Medicine Information 2019;42:157-8.

16. Bi P. Clinical efficacy observation on the treatment of mild acute pancreatitis (liver Qi stagnation type) with Yixianxiaoyan Decoction combining with Western medicine, Journal of Sichuan of Traditional Chinese Medicine 2019;(37):08138-41.

17. Yu F, Yang P, Wu Q. Effects of enteral and parenteral nutrition on proinflammatory factors and intestinal barrier function in severe acute pancreatitis. Journal of Modern Clinical Medicine 2016;42:131-3.

18. Sun $\mathrm{W}$, Chen $\mathrm{Y}, \mathrm{Li} \mathrm{H}$, et al. Clinical research progress of Analogous Presciption of Dachengqi Decoction in the treatment of acute pancreatitis. Chinese Journal of Experimental Traditional Medical Formulae 2019;12:221-6.

19. Stachecka J, Nowacka-Woszuk J, Kolodziejski PA, et al. The importance of the nuclear positioning of the PPARG gene for its expression during porcine in vitro adipogenesis. Chromosome Res 2019;27:271-84.

20. Du L, Yuan B, Zhang B, et al. Study on the mechanism of rhubarb's anti hyperlipidemia action based on the "auxiliary analysis system of the mechanism of action of traditional Chinese medicine". China Journal of Chinese Materia Medica 2015;40:3703-8.

21. Min L, Chen D, Qu L, et al. Tumor necrosis factor-a polymorphisms and colorectal cancer risk: a meta-analysis. PLoS One 2014;9:e85187.

22. Liu Y, Pang M. Overview on Dachaihu Decoction in the treatment of hyperlipidemia. Journal of Practical Traditional Chinese Internal Medicine 2017;06:86-9.

23. Zhang $Z$. Research progress in the pharmacological action of Poria cocos. Continuing Medical Education 2015;05:108-9.

24. Zhao Y, Niu K, Tang D, et al. Recent research on pharmacological action of peach kernel. Liaoning Zhongyi Zazhi 2015;04:888-90.

25. Wang Y, Wang Y, Ma Q, et al. Research progress of traditional Chinese medicine chicken's gizzard-membrane. Chinese Journal of Ethnomedicine and Ethnopharmacy 2014;19:10-2.

26. Li Q, Gan G, Liu Y. Research progress in chemical constituents and pharmacology of ligusticum chuanxiong hort. Shizhen Guoyi Guoyao 2006;07:1298-9.

27. Zhu L. Pharmacological analysis and clinical application analysis of safflower. Chinese Journal of Modern Drug Application 2016;16:286-7.

28. Tian T, Chen H, Feng Y, et al. Research progress on the pharmacology and toxicology of Alisma orientalis. Journal 
of Chinese Medicinal Materials 2014,11:2103-8.

29. Zhao Z, Zhang Q, Ji D, et al. Research progress on the pharmacological action of Hawthorn. Baokan Huicui 2017;02:112.

30. Yang X, Zhang K, Xu J, et al. Research progress on pharmacological action of peony bark. Journal of Henan University of Science and Technology (Medical Edition) 2012;02:157-8.

31. Xia T, Yang J, Liu Q, et al. Research progress on pharmacological action of polygonum cuspidatum. Zhejiang Journal of Integrated Traditional Chinese and

Cite this article as: Liu G, Liu F, Xiao L, Kuang Q, He X, Wang Y, Yu Y. Treatment of hyperlipidemic acute pancreatitis with modified Dachengqi decoction combining with conventional therapy based on "six-hollow-organs to be unblocked" theory. Ann Palliat Med 2020;9(4):2045-2053. doi: 10.21037/apm-20-1106
Western Medicine 2016;03:294-7.

32. Zhou B, Zhu S, Wang X, et al. Study on the mechanism of Qingxiajieyi formula in the treatment of severe pancreatitis. Shizhen Guoyi Guoyao 2012;23;220-1.

33. Liu Y. Forty cases of obesity and hyperlipidemia treated with Dachengqi Decoction combining with silkworm shit. Traditional Chinese Medicinal Research 2018;11:26-9.

34. Yao Du, Qin M, Cheng J. Observation on the effect of fenofibrate combining with low molecular weight heparin in the treatment of acute hyperlipidemic pancreatitis. Chinese and Foreign Medical Research 2018;16:162-4. 